\title{
Domestic energy management methodology for optimizing efficiency in Smart Grids
}

\author{
Albert Molderink, Vincent Bakker, Maurice G.C. Bosman, Johann L. Hurink, Gerard J.M. Smit
}

\begin{abstract}
Increasing energy prices and the greenhouse effect lead to more awareness of energy efficiency of electricity supply. During the last years, a lot of domestic technologies have been developed to improve this efficiency. These technologies on their own already improve the efficiency, but more can be gained by a combined management. Multiple optimization objectives can be used to improve the efficiency, from peak shaving and Virtual Power Plant (VPP) to adapting to fluctuating generation of wind turbines.

In this paper a generic management methology is proposed applicable for most domestic technologies, scenarios and optimization objectives. Both local scale optimization objectives (a single house) and global scale optimization objectives (multiple houses) can be used. Simulations of different scenarios show that both local and global objectives can be reached.
\end{abstract}

Index Terms-Smart Grid, Distributed Generation, Microgeneration, Energy, Demand Side Management

\section{INTRODUCTION}

Due to increasing energy prices and the greenhouse effect more efficient electricity production is desirable, preferably based on renewable sources. In the last years, a lot of technologies have been developed to improve the efficiency of the electricity usage and supply. One of the most eye catching technologies is generation based on renewable sources like large windturbine and photovoltaic (PV) parks. Also on domestic level a lot of technologies are in development. These technologies range from PV on roofs and micro Combined Heat and Power (microCHP) [1] upto controllable appliances [2]. The technologies can be subdivided in three groups:

- Distributed Generation (DG) In contrast to electricity generation in a few large power plants a growing share of the electricity is generated in smaller, geographically distributed generators. DG often has a higher efficiency or is based on renewable sources. Furthermore DG lowers transportation costs due to on site production. The DG generators range from windparks and bio-fuel plants on a megawatt level to domestic generators. Domestic generators are electricity generators on a kilowatt level placed in or nearby houses. The advantage of these domestic generators is that they are based on renewables sources (wind, sun) or they have a higher energy-efficiency (microCHP). These generators can produce heat and/or electricity.

- Distributed Electricity storage Especially with a growing amount of renewable sources in the electricity supply chain there is a growing demand for electricity storage

This research is conducted within the Islanded House project supported by E.ON Engineering.

All authors are with University of Twente, Department of Computer Science, Mathematics and Electrical Engineering, P.O. Box 217, 7500 AE, Enschede, The Netherlands,a.molderink@utwente.nl
[3]. Sometimes electricity can be produced more efficiently (e.g. at night) or only at certain times it is not needed (e.g. wind, sun) and thus needs to be stored. Large capacity electricity storage is difficult and has high losses, so distributed electricity storage may be a solution. In the MyGrid project hardware is developed to manage domestic electricity streams and store electricity within houses ${ }^{1}$.

- Demand side load management Large fluctuations in the load on the grid require fast adaptable but less efficient power plants (peak plants). Therefore, demand side load management can increase the generation efficiency by peak shaving [4] and by shifting load to more beneficial periods [5]. Parts of an appliance (e.g. the heating element of a dryer) can be temporarily switched off or the start of an appliance can be postponed [2]. About 50\% of the load in houses is dedicated to refridgerators, freezers, heaters, washing machines and dryers [6]. These appliances can be managed with only a little discomfort for the residents in contradiction to for example lights and a television. Field tests in the USA have shown that optimizatios with these appliances already can lead to significant peak reductions [2].

Altough all these three technologies already increase the energy efficiency, cooperation between the technologies and the existing infrastructure may lead to additional benefits. With an overall control algorithm optimizing the behavior of the domestic technologies even more efficiency can be gained. Such a control algorithm can have a local and a global scope. Within the local scope a single house is managed, within a global scope multiple houses are managed.

Next to increasing efficiency, a global control can also enhance the reliablity of supply [7], [8]. Various global controlling algorithms and products are developed both commercially 2 and from a more research point of view [6], [9], [10]. Three of these research algorithms are discussed in the next section in more detail.

Global controlling algorithms can have different optimization objectives, depending on the stakeholder of the global controller. A grid controller for example prefers to optimize the reliability of supply, a utility company prefers to balance demand and generation where the government likes to optimize efficiency. In general four different types of objectives can be identified:

- React on fluctuation of renewable sources A disadvantage of several renewable sources is that the generation is

\footnotetext{
${ }^{1}$ http://www.nedap-atrium.nl/, http://www.nedap-energysystems.com/

${ }^{2} \mathrm{http}: / /$ www.qurrent.com/
} 
less controllable and rather fluctuating, e.g. the electricity production of a windmill depends on the windspeed.

- Peak shaving High peaks in the demand to be supplied from the grid decrease the efficiency of supply and requires more generation capacity. These peaks can be lowered by global optimizations.

- Build Virtual Power Plants (VPP) A large number of microgenerators can deliver the same amount of electricity as a large power plant and can actually replace a power plant. An advantage of a VPP is that it can react faster on fluctuations and, therefore, can replace a peak plant or overcome generation/supply mismatches [11].

- Islanded operation/building microgrids The grid can be divided in clusters of a single houses (islanding) or streets, neighborhoods, cities, etc. (microgrids) [10], [12]. The energy production, usage and storage within these clusters can be balanced. Such a cluster then may become self-supporting or has only a small amount of import of electricity. The first option can be an ultimate goal for DG or is even necessary in case of a power outage. The second option can be part of the previous three objectives.

In this paper a local controlling algorithm is proposed to control domestic electricity and heat demand, as well as the generation and storage of heat and electricity. The algorithm divides the planning horizon into intervals and at the beginning of each interval a planning is made for that interval. This planning is based on the current state of the system and on the external condition on that moment (e.g. heat demand). The basic goal of this controller is to supply all heat and electricity demand without loss of comfort. Furthermore, the aim is to develop a local controller which is able to react on signals from a global controller to be able to support global optimizations. The combination of local and global controllers results in a Smart Grid [7] solution, controlling central power plants, non-domestic DG, non-domestic buffers and domestic imports/exports. Taking into account the continuous development of technologies mentioned above and different combinations of them in houses, the developed method has to be generic. To verify the quality of the controller, a simulator has been developped and used with various scenarios.

The remaining of this paper is structured as follows. The next section gives an overview of the relevant related work. In Section III a discription of the underlying model is given and Section IV presents the proposed control algorithm. Simulations of the algorithm are given in Section $\mathrm{V}$ and in Section VI the results are discussed. Section VII concludes this paper with a discussion and future work.

\section{RELATED WORK}

In [6] a market mechanism for energy allocation in microCHP grids is described. The grid is split up into Combined Heat and Power Micro Energy Grids (CHPMEG) that are loosely connected to the large-scale power grid. Within a CHPMEG producers and consumers trade their energy on a local market. The controllers of these CHPMEGs ensure that the prices are leveled within the large-scale power grid. Within the CHPMEG every producer gives its minimum energy price and every consumer its maximum bidding. The best combination of producers and consumers is determined by a mixed-integer optimization problem in every time interval. The energy storage is coupled to producers so they can deliver a part of their production to consumers and store the rest.

The Energy research Center of the Netherlands (ECN) proposes a Powermatcher in [9]. They expect a shift from the current central grid coordination to a distributed coordination. The Powermatcher is a multi-agent system with micro-economic principles resulting in a marked-based control concept for electricity supply and demand matching. Every producer, consumer and storage device is an agent. Supply/demand matching is organized in a hierarchical manner. To solve the problem of dependencies in production/consumption in consecutive time intervals they allow agents to bid not only for the next time interval, but also for (a limited number of) future intervals depending on whether they are allowed to produce/consume.

The Micro Grid Management System described in [10] is an agent based system for scheduling electricity production, storage and consumption with multiple types of agents. A micro grid is defined as a set of generators, storage devices and loads. Within a micro grid three types of agents are present: a source agent for every generator, a load agent for every load and a control agent. These agents themself are again built up by multiple agents. Based on forecasting schedules are created by the control agent. Based on these schedules it is decided which generators are switched on and which loads are supplied. A secondary control system controls and optimizes the electricity streams between micro grids.

The Gridwise Alliance [2] is a consortium of public and private stakeholders with a shared vision of a more efficient and cost effective energy generation, distribution and consumption. This consortium carried out a large scale test in Peninsula with their smart grid management system controlling DG and loads. Multiple technologies are used to control the generators and loads, for every type of generator and load a fitting technology is developed. Pricing signals on a local energy price market are used to steer the electricity production and consumption with an auction principle. The tests showed an average peak reduction of up to $30 \%$.

The main differences of these approaches with the proposed control method in this paper are the combination of heat and electricity, the partition in a global and a local controller, the generic solution for all types of domestic technologies and the use of mathematical optimization techniques.

\section{DOMESTIC ENERGY STREAMS}

The model of a single house is shown in Fig. 1. Every house consists of (several) micro-generators, heat and electricity buffers, appliances and a local controller. Multiple houses are combined into a grid, exchanging electricity and information between the houses.

Electricity can be imported from and exported into the grid. Heat is produced, stored and used only within the house. All domestic heat and electricity devices are divided into three groups:

- Producers produce electricity and/or heat. All available micro-generators are modelled in this way, considering 


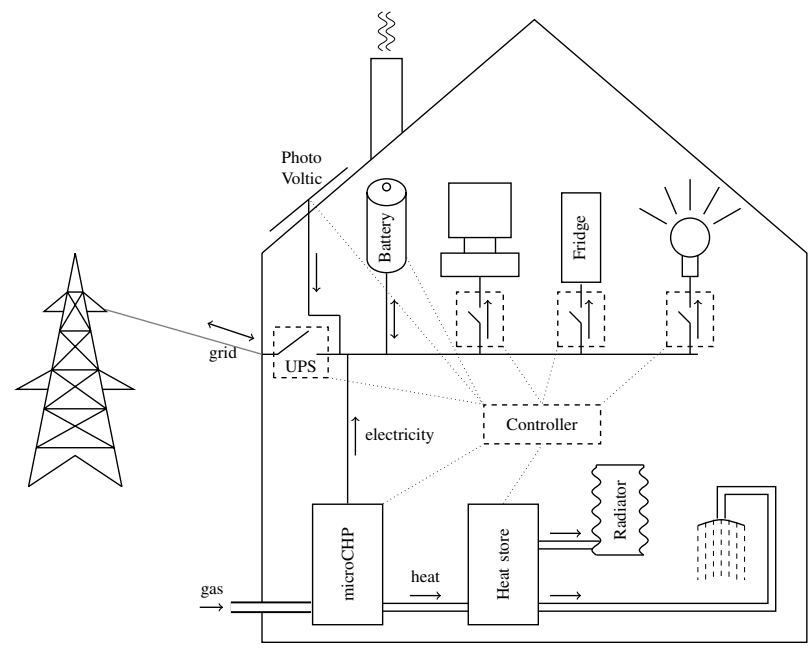

Fig. 1. Model of domestic energy streams

that the generation can be zero or even negative. A microCHP device produces electricity and heat, a Photo Voltaic produces only electricity where a conventional electric heater generates heat with a negative electricity production.

- Buffers store electricity and/or heat. When there is more energy production than consumption (and export) there is a surplus that flows into (one of) the buffers. A shortage (more consumption than production and import) flows out of (one of) the buffers.

- Consumers can consume electricity and/or heat. All consumers are modelled within this group, from fridges and coffeemakers to central heating and hot tapwater.

Every producer, buffer and consumer is called a device. Heat and electricity production is usually coupled on device level. For example some producers produce heat and electricity at the same time, hence production of heat and electricity are then coupled. A microCHP does either produce heat and electricity or nothing at all. The same holds for consuming devices, e.g. a hotfill washing machine. A more detailed description of the model can be found in [13].

Within the model, the planning horizon is discretisized resulting in a set of consecutive time intervals. The number of intervals depends on the length of the planning horizon and the length of the intervals.

\section{ENERGY MANAGEMENT METHODOLOGY}

The goal of the local energy management methodology is to make a generic solution for different (future) domestic technologies and house configurations. Furthermore, multiple scenarios should be possible, e.g. islanded scenario or houses connected to the grid. All these scenarios have to be covered by the methodology.

The primary functionality is to control the domestic generation and buffering technologies in such a way that they are used in a proper way and the heat and electricity supply is guaranteed for the house owners. The scheduling freedom of the domestic devices within these constraints can be used for optimizations. More scheduling freedom can be gained when residents are willing to decrease their comfort level. For example by accepting a temperature interval instead of a fixed temperature (scheduling freedom in heat production) or by accepting a deadline on the finish time of a washing machine instead of an exact starttime. This (small) decrease in comfort should lead to some benefits for the residents, e.g. by a reduced electricity bill.

Objectives can be local and global, thus the methodology should be generic for different local and global objectives. The global objectives are set by cost signals for electricity import and export sent by a global controller. The local controller has no knowledge of the global state but can react on steering signals from a global controller.

Summarising, a list of requirements for the energy management methodology is:

1) Device-level cost functions should include presents and future technologies.

2) Multiple scenarios with different objectives and costs for specific devices are possible.

3) Guaranteed comfort level chosen by the resident, given the incentives.

4) Both heat and electricity are considered and coupled to include combined heat/electricity producers and consumers.

5) Agreement between local and global controller by responding on steering signals and send status information.

6) Online scheduling possibilities for instantaneous matching of supply and demand and to respond on steering signals.

\section{A. Algorithm}

This subsection present an algorithm to control the devices in a single house. The algorithm is decomposed in time and is executed iterativly every time interval with parameters determined at the beginning of the time interval. Note that some of the parameters may be influenced by the solutions of former time intervals.

The first constraint for the algorithm is that the demand and supply within the house must match. The demand is defined as the sum of the heat and electricity demand of all consumers. This demand is given as an input parameter and can be matched with 1) import from the grid, 2) production by generators, 3) the buffers and 4) switching off consumers (not providing them). Switching off a device is seen as matching its demand (the demand is an input parameter and thus should not change). Every matching has a certain cost associated with it. When the sum of the four possibilities 1), 2), 3) and 4) gives more heat and/or electricity than the demand, the corresponding energy flows to a buffer and/or to the grid. Delivering energy to the grid or a buffer is seen as negative matching and leads to negative costs (reward).

Since every device in the house and the grid can match a certain amount of energy demand (optionally zero) for a certain cost and energy flowing to a buffer or the grid is seen as negative matching, all devices can be treated with the same definition of a cost function. Following to this setup, 


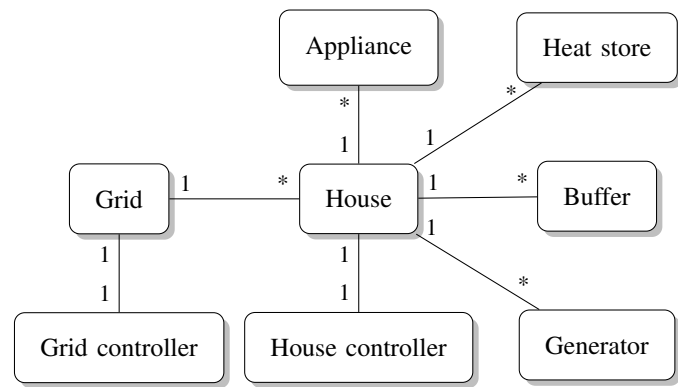

Fig. 2. Model used for the simulator

the algorithm has to find an optimal combination of matching sources within a homogeneous set of cost functions.

The cost functions for the devices are assumed to be generic to be able to apply the approach to different technologies. Furthermore, they have to be determined for every time interval seperately, since the production capacity, demand, buffer status and the costs can fluctuate over time. Finally, the global controller can send information about the cost function of the grid to the local controller as steering signals. Combining these local and global information, leads to a concrete cost functions in each time interval.

Within every time interval the control algorithm is ued on an optimization problem which is solved. The optimization problem considers a given set of devices Dev. For each device it is given how much it can match and for which costs. The goal is to find a mix of match sources with the lowest costs.

To model this problem, decission variables $x_{i}$ are introduced which express the amount of matching of device $i \in D e v$. These variables are used for both heat and electricity. However, since the ratio of matching heat and electricity is not always one, two multiplication factors are introduced, one for heat $\left(H_{i}\right)$ and one electricity $\left(E_{i}\right)$, e.g. the heat/electricity ratio of a microCHP is $8: 1$ thus $H_{i}=8$ and $E_{i}=1$.

The possible values for the variables $x_{i}$ are restricted, e.g. an consuming device is either switched on (in this case $x_{i}=0$ !) or switched off (in this case $x_{i}$ equals to demand of the consumer). Furthermore, the cost function parameters may rely on the concrete value of $x_{i}$. To model this, for each device $i \in D e v$ a set $S_{i}$ of intervals is specified and the variable $x_{i}$ is allowed to take only values from one of these disjoint intervals. Each interval $I_{i j}=\left[F_{i j}, T_{i j}\right] \in S_{i}$ specifies a uniform area for the variable $x_{i}$, in the sense that the costs associated with $x_{i} \in I_{i j}$ can be expressed by $A_{i j} \times x_{i}+B_{i j}$. The value $A_{i j}$ expresses the matching costs and $B_{i j}$ the startup costs if $x_{i}$ is chosen from the interval $I_{i j}$.

The problem of finding a best solution is modelled as an Integer Linear Program (ILP). The objective of the ILP is to minimize the costs while all given heat demand $D^{h}$ and electricity demand $D^{e}$ is matched. This is ensured with constraints in (3) and (4) given below. Furthermore, all values of $x_{i}$ must have a valid value. To ensure this, extra binary decision variables $c_{i j}$ are introduced and every $x_{i}$ is split up into variables $x_{i j}$ for every interval $I \in S_{i}$. With (5) is forced that for every device only one value of $c_{i j}$ is one. The variable $c_{i j}$ which gets one specifies the interval from which $x_{i}$ has to be chosen. Constraint (6) ensures this and leads to only one value of $x_{i j}$ to be nonzero for every device. The value of $x_{i}$ of a device gets defined as the sum of all $x_{i j}$ for that device (2).

$$
\begin{gathered}
\sum_{i, j} A_{i j} \times x_{i j}+c_{i j} \times B_{i j} \\
x_{i}=\sum_{j} x_{i j} \forall i \in D e v \\
D^{h}=\sum_{i} H_{i} \times x_{i} \\
D^{e}=\sum_{i} E_{i} \times x_{i} \\
\sum_{j} c_{i j}=1 \forall i \in D e v \\
c_{i j} \times F_{i j} \leq x_{i j} \leq c_{i j} \times T_{i j} \forall i \in D e v, j \in S_{i}
\end{gathered}
$$

\section{B. Determining costs}

The cost function of every device can express 1 ) the costs of the matching, 2) the costs of state transitions (e.g. startup costs) and 3) costs to steer the behaviour and reach some global objectives. Furthermore, the cost function of a device has to be independent of other devices to get a generic controller.

The matching costs are used to express what the costs of matching are for a certain device only concerning the status of the device itself. For example, the matching costs for electricity import are fixed and for microgenerators almost fixed. However, for buffers the costs fluctuate due to the filling rate. Since a lower filling rate leads to higher costs charging becomes more beneficial.

The costs for state transitions of the devices are used to prevent that costly transition happen too often or at undesirable times. For example, starting a microCHP leads to wearing, starting it often and with short intervals lead to extra wearing. Switching off a device shortly after starting it may even be forbidden (very high costs shortly after starting).

The global optimization objectives act mostly on the electricity import/export from the grid. To steer import/export, the costs of the import/export from the grid can be adjusted: higher costs when less import is preferred or costs depending on the amount of import.

In some scenarios it is desirable to allow a certain mismatch, e.g. during a power cut. In this scenario it is allowed to produce more heat than can be consumed/stored. This heat surplus can be dumped to enable the production of electricity (with a generator) when this is strictly necessary. Surplus energy can be added very easily by adding "abstract" devices for heat surplus/shortage and electricity surplus/shortage without changing the model. Two seperate devices for heat and electricity are used since heat surplus/shortage can be independent from electricity surplus/shortage. Using these devices in normal situation is prevented by defining extreme high costs for them, but this can be adapted in some circumstances. 


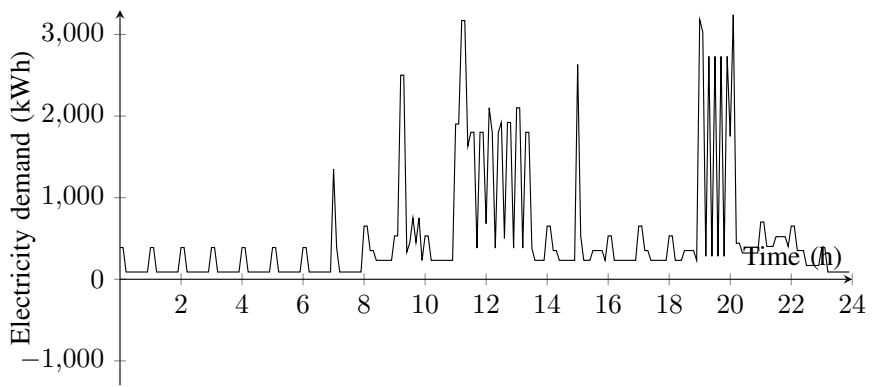

(a) Electricity demand

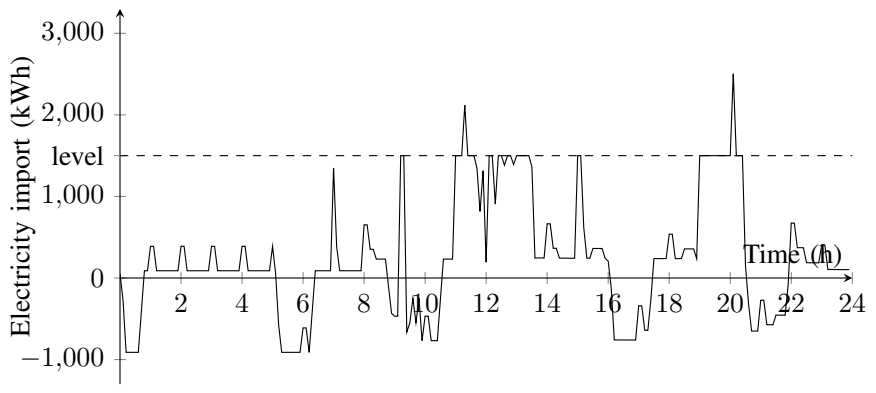

(c) Import in peak shaving scenario

Fig. 3. Electricity usage and imports for different scenarios

\section{Simulations}

Whether the optimizations in consecutive time intervals leads to the desired goals is verified by simulations. Especially the decomposition in time and the online scheduling of every time interval iterativly makes it hard to predict the results of the optimizations. The simulator used is developed at our research group to analyze different combinations of domestic producers, buffers, consumers and control algorithms.

The simulator is based on a model of a grid consisting of multiple houses connected to the grid importing and exporting electricity. Every house consists of producers, buffers and consumers. This model is simular to the model of the house described in section III. A schematic overview of the total model is given in Fig. 2.

The simulator is generic, thus other (new) technologies can added to the simulator. For every house is defined which generators, buffers and consumers are available. Every consumer starts consuming on a certain time (starttime), is switched on for a certain period (runtime) and has a electricity and heat usage profile. The controller can send signals to the producers and consumers to start and stop the devices.

\section{A. Scenarios}

Three scenarios are simulated to verify the proposed control methodology. Two of these scenarios are compared with normal operation where there is no objective but supplying the heat demand, just as with a normal thermostate (and heat buffer). The third scenario (islanded situation caused by a power outage) is compared with the results of a dedicated controller for such a situation described in [13].

The house used in the simulations consists of a microCHP, a Gledhill heatbuffer with $10 \mathrm{kWh}$ capacity and a battery with $1 \mathrm{kWh}$ capacity. Furthermore, consumers are added with a total

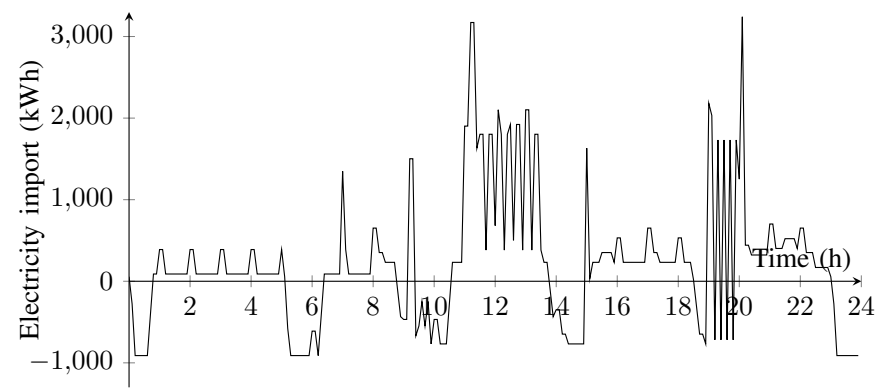

(b) Import during normal operation

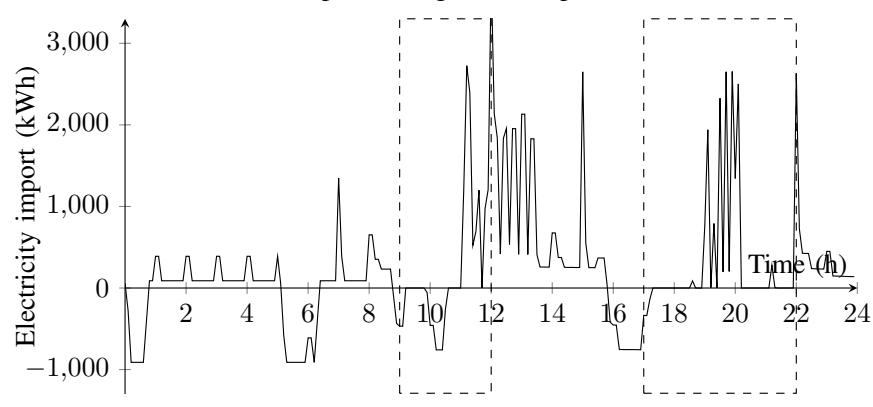

(d) Import with high import prices at 9.00-12.00 and 18.00-22.00

electricity consumption of $12.5 \mathrm{kWh}$ and heat consumption of $42 \mathrm{kWh}$ per day. The consumers consist of appliances present in most houses consuming on reasonable time periods. The overal total consumption and the profiles are based on measurements in normal houses and literature [14].

The scenarios studied are:

1) Reference scenario where the controller acts like a normal thermostate and microCHP controller. The battery is not used because there is no objective to be met.

2) Local optimizations using a microCHP and the battery to minimize peak imports.

3) Global optimizations using a microCHP and the battery to minimize imports on certain times of the day.

4) Power outage resulting in an islanded situation where the house has to supply its own heat and electricity. The microCHP and battery are used, but there are also consumers switched off and heat may be dumped.

\section{RESUlts}

The simulation results of the first three scenarios are given in Fig. 3. Subfig. 3(a) shows the electricity demand, the other three subfigures show the import from the grid. A negative import means an export to the grid. No smart appliances (ability to partly switch off) are used because no good models of these appliances are available and implemented yet. Furthermore, no appliances are switched off (except during the power outage). So the objectives should be reached by using the battery and shift the runtime of the microCHP while all heat and electricity is supplied and there is no energy shortage/surplus. In Subfig. 3(b) the import during normal operation, the first scenario without objective, can be seen. The battery is not used and the microCHP is switched on at the moment the heat buffer reaches the minimum level. 
Both the local and global objectives use the electricity import/export prices for optimization. For a local objective the electricity price is calculated based on the state of the house (generator statuses, buffer levels, demand, etc.) and the objective, for the global objective the electricity price is received from a global controller. Part of the local optimization can also be that the cost function of other parts are also changed. The results of the local optimizations, the objective to locally shave peaks above $1500 \mathrm{~W}$ import, are given in Fig. $3(\mathrm{c})$. It can be seen that $99 \%$ of the import is lower than 1500W.

Fig. 3(d) gives the results of global optimizations. The objective is to minimize imports during the periods 9.00-12.00 and 17.00-22.00, assuming that the electricity costs are higher in these periods. During the first part of the periods there is no import indicating that the objective is reached. However, during the second part of especially the second period the import is almost normal. This is caused by an empty electricity buffer and the fact that the heat buffer is filled during the first part and therefore the microCHP can not run anymore. After this period with normal imports the microCHP can be started and the import is again zero during the third part of the period.

The result of the Islanded scenario due to a power outage simulation is compared with the results of a dedicated islanded controller described in [13]. This dedicated controller is a proof of concept controlling only the electricity and not taking the heat demand, shortage and surplus into account. The goal of the simulations is verifying wether this generic controller can perform as good as a dedicated controller. The results of this simulation show that the generic controller perform slightly better on all criteria described in [13] for all scenarios. This is because the generic ILP algorithm bases the decision on the complete system state while the dedicated bases decision on a part of the system.

\section{DISCUSSION}

The simulation results show that a control of the devices based on objectives can be reached without energy shortage or surplus with most requirements (req) met. All heat and electricity demand is supplied without any discomfort for the residents (req 3 and 4). Altough only one configuration is tested, it may be assumed that the decission algorithm (ILP model) is generic for different configurations and techniques, since no knowledge of the configuration or techniques is incorperated in the model (req 1 and 2). Furthermore, the algorithm is executed every time interval during the planning interval (req 6). Finally, the controller is able to react on steering signals from the global controller but it can not yet communicate its scheduling freedom and shaving potential back to this controller in a generic way(req 5).

The most important drawback of this implementation of the controlling algorithm is that it decides based on the current status without taking the future into account. The best decision at this moment can have negative implications for future states while e.g. the second best choice may lead to an overall much better performance. Therefore, predictions of future states should be considered as additional input for the model.

\section{A. Future work}

To improve the algorithm, predictions of future states should be taken into account. This can be done by 1) extend the ILP by considering multiple time intervals instead of only one time interval or by 2) making a rough planning for a whole day based on predictions and use this planning while scheduling.

The complexity of solving an ILP is high, especially when multiple time intervals are considerd. Furthermore, it has to be solved every time interval. Therefore, a heuristic for solving the model has to be developed to decrease the complexity and to make it possible to implement the application in an embedded and energy efficient controller. The controller, the communication within the house and the additional hardware have to be energy-efficient, otherwise the control uses more energy than it saves.

Since the cost functions define the interaction between devices, cost functions must be adapted for every new configuration and scenario. To simplify determining the cost functions for an arbitrary technology and configuration, guidelines for the cost functions have to be obtained. These guidelines can also be used in a generic way to determine and communicate the scheduling freedom and shaving potential to the global controller.

\section{REFERENCES}

[1] United States Department of Energy, "The micro-CHP technologies roadmap," Results of the Micro-CHP Technologies Roadmap Workshop, December 2003.

[2] D. Hammerstrom, R. Ambrosio, T. Carlon, J. DeSteese, G. Horst, and R. Kajfasz, "Pacific northwest gridwise testbed demonstration projects, part i and ii," Pacific Northwest National Laboratory, July 2007.

[3] E. Furling, M. Piemontesi, P. Prasad, and D. Sukumar, "Advances in energy storage techniques for critical power systems," in The Battcon 2002 proceedings, 2002.

[4] A. Peacock and M. Newborough, "Controlling micro-chp systems to modulate electrical load profiles," Energy, vol. 32, no. 7, pp. 1093-1103, July 2007.

[5] M. Newborough and P. Augood, "Demand-side management opportunities for the uk domestic sector," in IEE Proceedings Generation, Transmission and Distribution, vol. 3, May 1999, pp. 283-293.

[6] C. Block, D. Neumann, and C. Weinhardt, "A market mechanism for energy allocation in micro-chp grids," in 41st Hawaii International Conference on System Sciences, Jan 2008, pp. 172-180.

[7] J. Scott, P. Vaessen, and F. Verheij, "Reflections on smart grids for the future," Dutch Ministry of Economic Affairs, Apr 2008.

[8] "Distributed generation in liberalised electricity markets," 2002.

[9] J. Kok, C. Warmer, and I. Kamphuis, "Powermatcher: Multiagent control in the electricity infrastructure," in 4th international joint conference on Autonomous agents and multiagent systems. ACM, Jul 2005, pp. 75-82.

[10] J. Oyarzabal, J. Jimeno, J. Ruela, A. Englar, and C. Hardt, "Agent based micro grid management systems," in Internation conference on Future Power Systems 2005. IEEE, Nov 2005, pp. 6-11.

[11] V. Bakker, A. Molderink, J. Hurink, and G. Smit, "Domestic heat demand prediction using neural networks," in 19th International Conference on System Engineering. IEEE, 2008, pp. 389-403.

[12] S. Abu-sharkh, R. Arnold, J. Kohler, R. Li, T. Markvart, J. Ross, K. Steemers, P. Wilson, and R. Yao, "Can microgrids make a major contribution to uk energy supply?" Renewable and Sustainable Energy Reviews, vol. 10, no. 2, pp. 78-127, Sept 2004.

[13] A. Molderink, V. Bakker, J. Hurink, and G. Smit, "Algorithms for balancing demand-side load and micro-generation in islanded operation," in 19th International Conference on System Engineering. IEEE, 2008, pp. 389-403.

[14] A. Wright and S. Firth, "The nature of domestic electricity-loads and effects of time averaging on statistics and on-site generation calculations," Applied Energy, vol. 84, no. 4, pp. 389-403, April 2007.

\section{BIOPGRAPHIES}




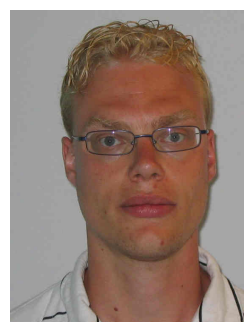

Albert Molderink was born in Heerenveen (The Netherlands) in 1983. He received his B.Sc and M.Sc. degree in Computer Science from the University of Twente, Enschede, The Netherlands, in respectively 2004 and 2007. In addition, he received a Electrical Engineering minor certificate. When he completed his study he started working towards a $\mathrm{Ph} . \mathrm{D}$. degree at the University of Twente under supervision of Prof. dr. ir. G.J.M. Smit of the Computer Architecture of Embedded Systems (CAES) group, faculty of Electrical Engineering, Mathematic and Computer Science. He is working in a research group that investigates the possibilities of increasing energy efficiency using embedded control, mainly via optimization and control algorithms. His research focus is on algorithms to optimize energy streams within a house. His research interests include energy efficiency, mathematical modelling and optimizations, algorithm development and embedded hardware. 